\title{
Respectful maternity care for positive birthing experience at Pravara Rural Hospital
}

\author{
Vidyadhar B. Bangal ${ }^{1 *}$, Sangita Vikhe², Shobha Borhade², Ujjwala Thorat $^{1}$
}

\begin{abstract}
${ }^{1}$ Department of Obstetrics and Gynecology, Rural Medical College, Pravara Institute of Medical Sciences (Deemed to be University) Loni, Ahmednagar, Maharashtra, India

${ }^{2}$ Department of Nursing, Pravara Rural Hospital, Pravara Institute of Medical Sciences (Deemed to be University) Loni, Ahmednagar, Maharashtra, India
\end{abstract}

Received: 27 May 2020

Accepted: 03 July 2020

\section{*Correspondence:}

Dr. Vidyadhar B. Bangal,

E-mail: smritijainagrawal@ rediffmail.com

Copyright: ( ) the author(s), publisher and licensee Medip Academy. This is an open-access article distributed under the terms of the Creative Commons Attribution Non-Commercial License, which permits unrestricted non-commercial use, distribution, and reproduction in any medium, provided the original work is properly cited.

\section{ABSTRACT}

Background: Many women in developing countries experience disrespect and abuse during labour and delivery. Respectful maternity care (RMC) is considered as one of the basic reproductive health rights of the women. It is one of the essential components of LaQshya programme of Government of India. The aim of the study was to highlight the important components of the RMC, its implementation and its impact on patient turnover in the maternity unit of Pravara Rural Hospital Loni and review the literature on the subject.

Methods: A prospective observational study was conducted for a period of one year from January 2019 to December 2019 at tertiary care hospital. The implementation of RMC was observed and important findings were documented. The patient turnover and cliental satisfaction was noted.

Results: It was observed that all components of RMC were strictly followed in maternity unit of Pravara Rural Hospital Loni. The staff and doctors were trained and oriented towards importance of RMC. The patient turnover has increased exponentially year by year. The patient feedback system about the quality of care in labour and delivery ward shows overall satisfaction score of 4.3 on the 5-point Likert scale. There was a surveillance system that supervises and closely monitor the quality of care in labour room in general and RMC in particular.

Conclusions: RMC is one of the important components of LaQshya certification process. Respectful maternity care is implemented at Pravara Rural hospital in its true spirit. It has resulted in gaining the faith and trust of the community, which is reflected through exponential rise in the number of deliveries taking place in the hospital.

Keywords: LaQshya certification, Reproductive health rights of women, Respectful maternity care

\section{INTRODUCTION}

In every country and community around the world, pregnancy and childbirth are hugely important events in the lives of women and families. They are also a time of great vulnerability. The relationship with maternity caregivers and the maternity care system during this time is incredibly important. ${ }^{1}$ Positive birthing experience is considered as the universal right of childbearing women. The issue of disrespect and abuse during childbirth may seem simple on the surface. However, it is deeply rooted in complexity. It is not specific to South Asia, or subSaharan Africa, but rather disrespect and abuse are global issues that affect every health system in the world. ${ }^{2}$

Several studies have sought to quantitatively measure the extent of disrespect and abuse during childbirth. These studies show that disrespect and abuse of women during childbirth are prevalent, although the estimates varied widely (from $15 \%$ to $98 \%$ ) because of several methodological issues, including inconsistencies in how disrespect and abuse were measured. ${ }^{3}$ There is suggestive 
evidence that disrespect and abuse deter women from utilizing institutional health services. ${ }^{4}$

The concept of "safe motherhood" is usually restricted to physical safety, but childbearing is also an important rite of passage, with deep personal and cultural significance for a woman and her family. Because motherhood is specific to women, issues of gender equity and gender violence are also at the core of maternity care. Thus, the notion of safe motherhood must be expanded beyond the prevention of morbidity or mortality to encompass respect for women's basic human rights, including respect for women's autonomy, dignity, feelings, choices, and preferences, including companionship during maternity care. ${ }^{5}$

The paper highlights the importance of respectful maternity care and explain the labour room protocols that are followed and the experience regarding implementation of "respectful maternity care" at Pravara Rural Hospital, Loni.

\section{METHODS}

A prospective observational study was conducted for a period of one year from January 2019 to December 2019 in the department of obstetrics and gynecology of 1275 bedded tertiary care teaching hospital. Approximately 10,000 deliveries take place annually in this hospital. Fifty percent delivery cases are un-booked and they directly report to labour room. Majority of them are registered at either public health facilities or private facilities during antenatal period. Respectful maternity care (RMC) is practiced in the maternity unit since its inception.

The labour room protocol that is followed at Pravara Rural Hospital is as follows. Women are received in a triage room and are then segregated according to the assessment. Women who are admitted to labour delivery complex remain inside till they deliver. They are closely observed for occurrence of any complication for two hours of postpartum. Normal and low risk deliveries are conducted either by resident doctors or the senior trained nurses. All high-risk deliveries, instrumental and operative deliveries are conducted by either resident doctors or the consultant obstetrician. All nursing staff, doctors, support staff are trained and oriented towards the components of the RMC. The labour companion is chosen by woman herself, thus her autonomy is preserved by allowing her to decide, who she wants to be with during the course of labour. The chosen companion is explained about her role and responsibilities, before she is permitted in the labour delivery room complex. As a part of hospital policy, male members of the family are not allowed in the labour room.

The labour companion remains with laboring woman throughout the labour process including second stage. The labour companion is asked to encourage woman during bearing down in second stage and keep patience during the process of labour. The labour companion is counselled and informed, if any labour abnormality is detected, that requires medical or operative intervention in the form of instrumental vaginal delivery or caesarean section. The labour companion helps women during early initiation of breast feeding and support her during early ambulation and moving out of bed and for accompanying to wash room.

\section{RESULTS}

The quality of services of the obstetric unit of Pravara Rural Hospital has gained the faith and trust of the community, which is reflected through continuous rise in patient turnover in labour ward every year (Figure 1).

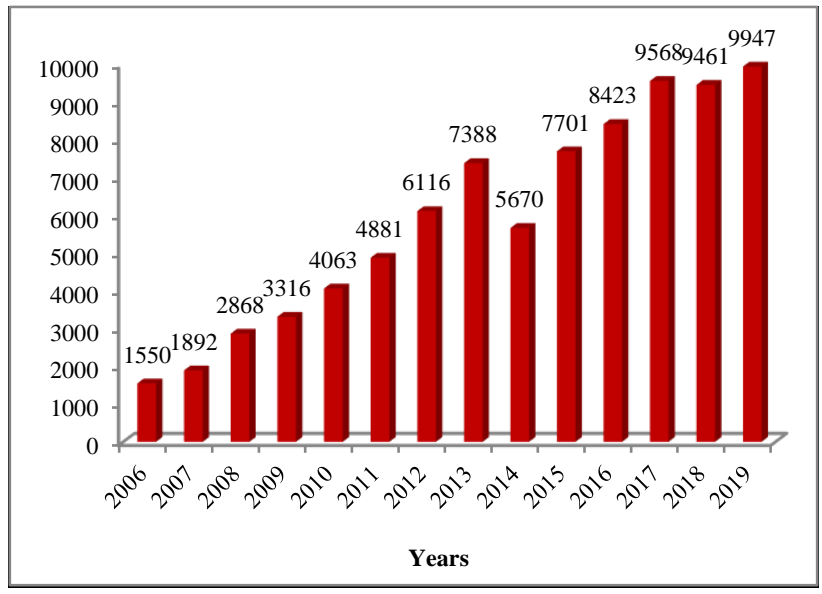

Figure 1: Steady rise in number of deliveries at Pravara Rural Hospital (2006-2019).

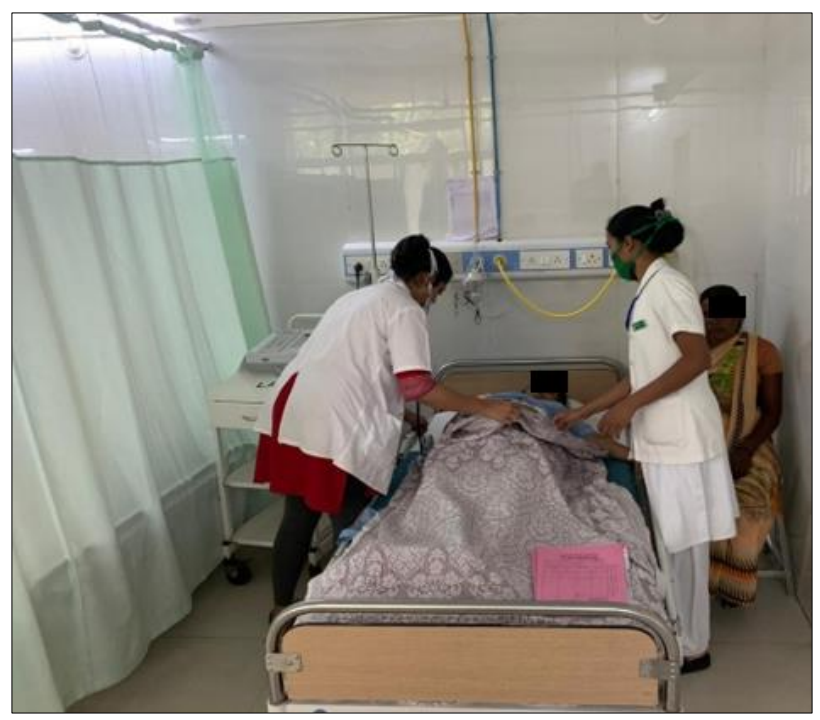

Figure 2: Good ambience, privacy and personalized attention to woman in labour.

The good infrastructure, ambience, privacy, cleanliness, state of art facilities, bi-directional communication, have been the hall mark of maternity services of Pravara Rural 
hospital Loni (Figure 2). The labour room nursing staff provide individual attention and support to woman during early postnatal period for early initiation of breast feeding (Figure 3). The concept of labour companion is implemented and is proving helpful (Figure 4).

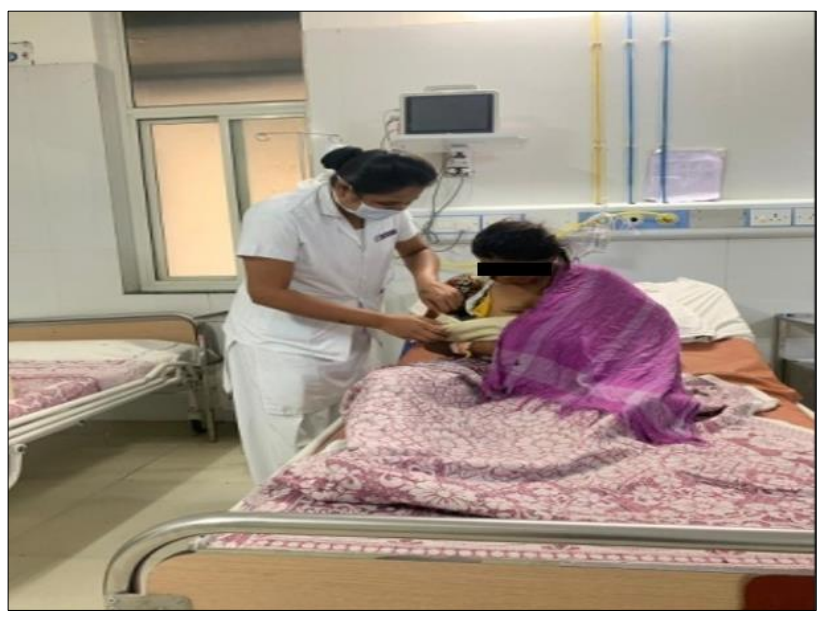

Figure 3: Care and support to postnatal woman for early initiation of breast feeding.

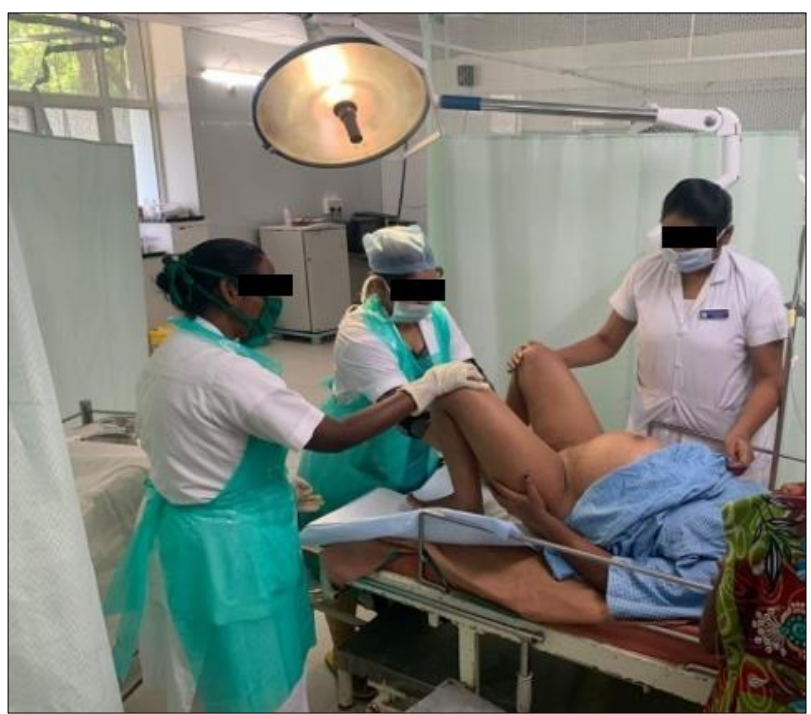

Figure 4: Availability of labour companion at the time of delivery.

There is improved satisfaction about the quality of care among the parturient and relatives. The labour companion has reduced the need of nursing staff for fulfilling the physical and emotional requirements of the women in labour. Nursing staff are able to utilize the saved time for care of other women. It has become easy to counsel the woman and the relatives about the progress of labour and in case there is need for surgical intervention like instrumental vaginal delivery or caesarean section. The epidural labour analgesia facility is made available for desiring women. The epidural facility has helped to reduce caesarean sections due to tokophobia. The issue of baby sex identification at birth and the baby handing over to relatives has been streamlined, as the relative is available at the time of labour. There is a system of incidence reporting and feedback from the women and the relatives about the quality of services. The patient feedback system about the quality of care in labour and delivery ward shows overall satisfaction score of 4.3 on the 5-point Likert scale. There is a surveillance mechanism that monitors the quality of care. The system takes care of the complaints received from the beneficiaries and prompt inquiry in to the matter. Overwork, stress, lack of sleep among nurses and doctors have been observed as predisposing factors for occasional incidences of deviation from the norms of RMC. At times, the women in labour are not co-operative for normal process of labour or insist for certain unnecessary interventions during labour.

\section{DISCUSSION}

The quality of childbirth care in facilities, including women's, newborns' and families' experience of this care, is an important determinant of families' decision to use, or not to use, facility childbirth services. ${ }^{6-8}$ In many countries, women experience mistreatment during childbirth and are unable to make choices that put them in control of their own experience. 6,9

Disrespect or verbal or physical abuse during labour is a problem that doesn't belong to one country, to one region of the world, to one income level or to one particular population. For the same reason, all over the world, maternal health efforts are shifting from an emphasis on improving quality of care. The researchers and policy makers started working and giving importance to the issue of the treatment women receive during the course of labour and delivery at health care facilities. ${ }^{6}$

The global attention was drawn in year 2010 by Bowser and Hill's landscape analysis. ${ }^{10}$ Many studies have documented the nature of disrespectful care and its adverse effects on care-seeking behaviour, 2 and calls to action on quality of maternal health care have prioritized women's experiences. Before 2015, most of the research on women's experiences during childbirth was qualitative in nature. ${ }^{11}$

According to Hill, there is suggestive evidence that disrespect and abuse deter women from utilizing institutional health services. In countries where traditional, often unskilled, health services offer a culturally acceptable alternative to the formal health sector, such mistreatment impedes the delivery of skilled birth services to women. Some nurses rough you up to the extent that you tell her to let you deliver alone, one Kenyan woman told Hill. You are in pain and all she does is give you a harsh and rude approach. That is why I don't go to the hospital to deliver, because I am not used to somebody who roughs me up. However, Hill cautioned against an oversimplified explanation of poor service, noting that abuse often arises when providers and staff 
are themselves feeling overwhelmed by workforce shortages, scarcities of essential supplies or a lack of promotional opportunities. How do you expect a midwife to be in a good mood if she works with no breaks and has many clients to attend to in a dirty working environment? asked Warren. These conditions lay the groundwork for disrespect and help explain why patient neglect is so prevalent, particularly in countries that lack enforceable national laws and oversight over health services, said Hill. One Kenyan nurse told Hill, by the ninth, tenth, eleventh delivery of the night, I would have been rated minus zero. If you care for the nurses, they will care for the patients.

Warren reported that women were consistently subjected to non-confidential, non-consensual and non-dignified care. For example, Kenyan women recalled being scolded for screaming during childbirth, slapped by the medical staff and forced to walk around the ward naked. Further, only $38 \%$ of providers responded that women have the right to be informed of the procedures being performed and only $37 \%$ responded that information confidentiality was important. ${ }^{10}$

Bohren and colleagues' study extends the evidence that many women are mistreated during facility-based childbirth in low-resource settings. In addition, other studies-including a birth observation study in five countries (Ethiopia, Kenya, Madagascar, Rwanda, and Tanzania) 6 and more recently another study that used a validated person-centred maternity care scale in surveys with women in Kenya, Ghana, and India, have highlighted that the problem is not just about the presence of negative interactions such as verbal and physical abuse but also an absence of positive interactions such as effective communication and supportive care. ${ }^{12,15}$

Furthermore, studies in high-income countries have shown that mistreatment is not just an issue in lowresource settings. ${ }^{13,14}$ Bohren and colleagues' study also extends the evidence on disparities in how women are treated on the basis of age and socioeconomic status. Sources of disparities highlighted in other studies have included the type of facilities women receive care in and race and ethnicity. ${ }^{6}$

Freedman and Kruk define disrespect and abuse during childbirth as interactions or facility conditions that local consensus deem to be humiliating or undignified, and those interactions or conditions that are experienced as or intended to be humiliating or undignified. ${ }^{15}$ Building off of this definition and a 2010 landscape analysis by Bowser and Hill, Bohren et al outlined 7 categories of disrespectful and abusive care during childbirth: (1) physical abuse; (2) sexual abuse; (3) verbal abuse; (4) stigma and discrimination; (5) failure to meet professional standards of care; (6) poor rapport between women and providers; and (7) health system conditions and constraints. ${ }^{6,16}$ These categories were subsequently conceptualized in 2 dimensions: (1) intentional use of violence, including physical abuse, verbal abuse, and negligent withholding of care and (2) structural disrespect, which stems from deviations from accepted standards for infrastructure, staffing, equipment availability, and supplies needed to deliver care, as well as in unnecessary interventions, demands for illegal payments, and the detainment of people in facilities until they have paid their bills. ${ }^{17}$

In rural area, women are illiterate and belong to lower socio-economic class. They are not aware about the reproductive health rights and are thus more tolerant towards and disrespect and abusive treatment they receive at health facilities. In urban areas, with growing literacy and women empowerment, women have started expressing their concerns, if get exposed to disrespectful treatment. The hospital managers are now aware about the importance of cliental satisfaction, and more than that, about the dis-satisfaction which can affect the hospital reputation and patient turn over. Hospitals also face the consequences of the ethical and legal issues which can crop up.

In addition to bringing vital, possibly life-saving health services, women's experiences with maternity caregivers have the power to give strength and comfort or to cause lasting damage and emotional trauma. Either way, women's memories of their childbearing experiences stay with them for a lifetime. A "veil of silence" has covered up the humiliation and abuse suffered by women seeking maternity care. In addition, to date there is no agreement on what respectful maternity care means. It is clear, however, when authors look at international human rights standards, that disrespect and abuse is a violation of women's basic human rights. The concept of safe motherhood is usually restricted to physical safety, but safe motherhood is more than just the prevention of death and disability. It is respect for women's basic human rights, including respect for women's autonomy, dignity, feelings, choices, and preferences, including companionship during maternity care.

Recent studies illustrate this fact: a recent populationbased study in Tanzania by Kruk and colleagues that examined women's choices showed that "provider attitude" was the highest predictor, along with availability of commodities, of women's choice to use facility-based childbirth services. It mattered to women more than cost, distance, and lack of availability of free transport. This suggests that provider attitude is important in determining whether or not women deliver in facilities with skilled providers. ${ }^{7}$

\section{CONCLUSION}

All childbearing women need and deserve respectful care and protection of their autonomy and right to selfdetermination; this includes special care to protect the mother-baby pair as well as women in a context of marginalization or heightened vulnerability (e.g., 
adolescents, ethnic minorities, and women living with physical or mental disabilities or HIV). Pravara Rural Hospital offers a relationship characterized by caring, empathy, support, trust, confidence, and empowerment, as well as gentle, respectful, and effective communication to enable informed decision making. Authors firmly believe that disrespect and abuse during maternity care are a violation of women's basic human rights. Respectful maternity care must be supported in totality at all levels of health facilities.

Funding: No funding sources

Conflict of interest: None declared

Ethical approval: The study was approved by the Institutional Ethics Committee

\section{REFERENCES}

1. The White Ribbon Alliance. Washington, DC: The White Ribbon Alliance for Safe Motherhood; Respectful maternity care: the universal rights of childbearing women. 2011. Available at: https://www.whiteribbonalliance.org/wpcontent/uploads/2017/11/Final_RMC_Charter.pdf website. Accessed 20 April 2020.

2. Family Care International: Care-seeking during pregnancy, delivery and the postpartum period: a study in Homa Bay and Migori districts, Kenya. 2005, New York: FCI. The Skilled Care Initiative Technical Brief: Compassionate Maternity Care: Provider Communication and Counselling Skills; 2005.

3. Miller S, Abalos E, Chamillard M, Ciapponi A, Colaci D, Comandé D, et al. Beyond too little, too late and too much, too soon: a pathway towards evidence-based, respectful maternity care worldwide. Lancet. 2016;388:2176-92.

4. Kujawski S, Mbaruku G, Freedman LP, Ramsey K. Association between disrespect and abuse during childbirth and women's confidence in health facilities in Tanzania. Maternal Child Health J. 2015;19(10):2243-50.

5. Respectful Maternity Care. Available at: http://www.whiteribbonalliance.org/index.cfm/actnow/respectful-maternity-care/). Accessed 14 May 2020.

6. Bohren MA, Vogel J, Hunter E, Lutsiv O, Makh S, Souza J, Aguiar C, et al. The mistreatment of women during childbirth in health facilities globally: A mixed-methods systematic review. PLoS Med. 2015;12(6):e1001847.

7. Kruk ME, Kujawski S, Mbaruku G, Ramsey K, Moyo W, Freedman LP. Disrespectful and abusive treatment during facility delivery in Tanzania: a facility and community survey. Health Policy and Planning. 2018;33(1):e26-33.

8. Abuya T, Warren CE, Miller N, Njuki R, Ndwiga C, Maranga A, Mbehero F, et al. Exploring the prevalence of disrespect and abuse during childbirth in Kenya. PloS One. 2015;10(4):e0123606.

9. Sen G, Reddy B, Iyer A. Beyond measurement: the drivers of disrespect and abuse in obstetric care. Reprod Health Matters. 2018;26:6-18.

10. Bowser D, Hill K. Exploring evidence for disrespect and abuse in facility-based childbirth: report of a landscape analysis. USAID/TRAction Project; 2010.

11. Sando D, Abuya T, Asefa A, Banks KP, Freedman LP, Kujawski S, et al. Methods used in prevalence studies of disrespect and abuse during facility based childbirth: lessons learned. Reprod Health. 2017;14(1):1-8.

12. Rosen HE, Lynam PF, Carr C, Reis V, Ricca J, Bazant ES, et al. Direct observation of respectful maternity care in five countries: a cross-sectional study of health facilities in East and Southern Africa. BMC Preg Childbirth. 2015;15(1):306.

13. Afulani PA, Phillips B, Aborigo RA, Moyer CA. Person-centred maternity care in low-income and middle-income countries: analysis of data from Kenya, Ghana, and India. Lancet Glob Health. 2019;7:e96-e109.

14. Vedam S, Stoll K, Taiwo TK, Rubashkin N, Cheyney M, Strauss N, et al. The Giving Voice to Mothers Study: inequity and mistreatment during pregnancy and childbirth in the United States. Reprod Health. 2019;16:77.

15. Freedman L, Ramsey K, Abuya T, Bellows B, Ndwiga C, Warren CE, et al. Defining disrespect and abuse of women in childbirth: a research, policy and rights agenda. Bull World Health Organ. 2014;92(12):915-7.

16. Browser D, Hill K. Exploring Evidence for Disrespect and Abuse in Facility-Based Childbirth: Report of a Landscape Analysis-USAID. Available at: https://www.ghdonline.org/uploads/Respectful_Care _at_Birth_9-20-101_Final1.pdf. Accessed 20 April 2020.

17. Jewkes R, Penn-Kekana L. Mistreatment of women in childbirth: time for action on this important dimension of violence against women. PLoS Med. 2015;12(6):e1001849.

Cite this article as: Bangal VB, Vikhe $\mathrm{S}$, Borhade $\mathrm{S}$, Thorat U. Respectful maternity care for positive birthing experience at Pravara Rural Hospital. Int J Reprod Contracept Obstet Gynecol 2020;9:3297-301. 